\title{
Aquatic versus land-based exercise for cardiorespiratory endurance and quality of life in obese patients with knee osteoarthritis: a randomized controlled trial
}

\author{
Tirza Z. Tamin, Natalia Loekito
}

Department of Physical Medicine and Rehabilitation, Faculty of Medicine, Universitas Indonesia, Cipto Mangunkusumo Hospital, Jakarta, Indonesia

\section{ABSTRACT}

Background: Obesity with knee osteoarthritis $(\mathrm{OA})$ is related to chronic pain causing physical inactivity that leads to decreased cardiorespiratory endurance and quality of life. Aquatic and land-based exercises are effective in improving physical activity. The aim of this study is to compare between aquatic and land-based exercise to improve cardiorespiratory endurance and quality of life in obese patients with knee osteoarthritis.

Methods: A single-blind, randomized, controlled trial was conducted on thirty-three obese patients with knee OA who visited Obesity Clinic of Medical Rehabilitation Cipto Mangunkusumo Hospital, from October 2016 to January 2017, subjects were divided into aquatic or land-based exercise group. Aerobic and knee-strengthening exercises were given. Cardiorespiratory endurance was assessed using the Borg Scale, whereas both the BORG CR-10 and SF-36 questionnaires were used to assess quality of life.

Results: After intervention, there were significant improvements in the land-based exercise group in the rating of perceived exertion $(\mathrm{p}=0.02)$, role limitations due to physical health $(\mathrm{p}=0.024)$, role limitations due to emotional problems $(\mathrm{p}=0.041)$, energy/fatigue $(p=0.016)$, and the decline in pain $(p=0.049)$ parameters. While in the aquatic exercise, there were significant improvements in leg fatigue ( $p=0.016)$, energy/fatigue $(p=0.025)$, emotional well-being $(p<0.001)$, and general health $(p=0.045)$ parameters. Despite this, there were no significant differences between two groups regarding cardiorespiratory endurance and quality of life.

Conclusion: This study found that patients could start aquatic exercise to reduce leg fatigue and enhance general health and energy. After that, exercise could be continued in land-based settings to improve cardiorespiratory endurance and quality of life.

Keywords: aquatic exercise, cardiorespiratory endurance, knee osteoarthritis, land-based exercise, obesity, quality of life

pISSN: 0853-1773• eISSN: 2252-8083• https://doi.org/10.13181/mji.v27i4.2107• Med J Indones. 2018;27:284-92

- Received 07 Jul 2017 • Accepted 06 May 2018

Corresponding author: Tirza Z. Tamin

tirzaediva.tamin@gmail.com

Copyright @ 2018 Authors. This is an open access article distributed under the terms of the Creative Commons Attribution-NonCommercial 4.0 International License (http://creativecommons.org/licenses/by-nc/4.0/), which permits unrestricted non-commercial use, distribution, and reproduction in any medium, provided the original author and source are properly cited. 
The prevalence of obesity has increased dramatically around the world. It is estimated that the prevalence of obesity in 2025 will increase to $50 \%$ in developed countries. ${ }^{1}$ Riset kesehatan dasar (RISKESDAS) 2013 showed percentage of the population in Indonesia with obesity continues to increase from year to year. DKI Jakarta is one of the provinces with higher prevalence than the national prevalence. ${ }^{2}$ Data from the Obesity Outpatient Clinic of Medical Rehabilitation Department in Cipto Mangunkusumo Hospital showed an increase in the number of visitors from 1,484 in 2013 to 1,633 in 2015.

The risk of osteoarthritis $(\mathrm{OA})$ is increased in obese people caused by weight load and associated with the activation of an inflammatory pathway, which leads to synovial pathology. ${ }^{1}$ The Framingham study showed that weight loss is associated with decreased risk of both onset and progression of OA. This finding means that in women, a reduction of body weight by 10 pounds $( \pm 5 \mathrm{~kg}$ ) over ten years would lower the risk of symptomatic knee OA by more than $50 \%{ }^{3}$

Exercise as an obesity management strategy has been shown to be beneficial for weight loss. ${ }^{4}$ Walking, running, or cycling in the land-based is common modes of the exercise programs which have negative problems such as increased muscle pain and risk of musculoskeletal injuries due to accumulated stress on lower-joint extremities, which reduces adherence to exercise training. ${ }^{5,6}$ Aquatic exercise can involve whole-body movements which forces increases heart rate and energy expenditure because water is a comparatively high-density medium (12 times more resistant than air). ${ }^{6}$ Aquatic exercise has also been shown to support body weight to produce less musculoskeletal stress. Buoyancy and the hydrostatic pressure of water can be both beneficial and enjoyable for clinical or obese populations. Furthermore, because of water's higher specific heat and thermoregulatory characteristics, permitting training intensity or duration to progress at a greater rate than with land-based exercises. This suggests that aquatic exercise might be perceived as comparatively less strenuous and might serve as an ideal method to improve the functional health status and reduce body weight of obese individuals or for elderly population who has age-related degenerative changes. ${ }^{6-8}$
Based on the information above, we were encouraged to conduct a study on exercise programs for cardiorespiratory endurance and quality of life in obese patients with knee $\mathrm{OA}$ as the study has never been done before, especially in Indonesia. The aim was to observe whether there is a difference in cardiorespiratory endurance and quality of life improvement in patients who were given aquatic exercise compared with those who received land-based exercise.

\section{METHODS}

In this study, a single-blind, randomized, controlled trial was used in obese patients with knee OA who visited the Obesity Outpatient Clinic and Musculoskeletal Division of the Medical Rehabilitation Department of Cipto Mangunkusumo Hospital from October 2016 to January 2017.

\section{Subjects}

The sample size was estimated based on previous studies (pilot study) calculating using a formula for a difference of cardiorespiratory endurance and quality of life between two independent groups. This calculation obtained a minimum of sample size should be 24 subjects for each group. However, based on our calculations, we sum up to 57 subjects to deal with dropout effects. Samples were obtained by consecutive sampling.

The inclusion criteria in this study were: 1 .) men and women aged $40-80$ years, 2 .) height $145-170 \mathrm{~cm}, 3$.) obese people (grade I if body mass index (BMI) $>25 \mathrm{~kg} / \mathrm{m}^{2}$ but $<30 \mathrm{~kg} /$ $\mathrm{m}^{2}$, grade II if BMI $\geq 30 \mathrm{~kg} / \mathrm{m}^{2}$ ) with knee OA grades 2 and 3 based on the Kellgren-Lawrence grading scale, 4.) pain intensity visual analog scale (VAS) 4-6, and 5.) sedentary lifestyle (physical activity level between 1.4 and 1.69). We excluded patients with visual and hearing impairment, cognitive impairment (mini mental state exam $\leq 23$ ), and any musculoskeletal (joint deformity), neuromuscular, metabolic, and cardiopulmonary disease that would interfere the intervention. The use of lower extremity orthoses and those with contraindications to hydrotherapy (such as skin disease, infectious disease, fever, uncontrolled blood pressure, and seizure history, tracheostomy, stoma, urine, and fecal incontinence) were also excluded. 
All subjects who agreed to the intervention were informed about study procedure, it risks and side effects and gave written informed consent. Participants were considered dropouts when they missed three consecutive exercise sessions in one week, had worsening clinical conditions, and withdrew from the research.

\section{Randomization}

Before the start of the study, lists of subjects were randomized using a simple random sampling method, in which selection was made randomly using a computer. Subjects were divided into two groups, aquatic exercise and land-based exercise.

\section{Aquatic exercise and land-based exercise}

Aerobic and strengthening exercises were given as interventions in this study. In landbased exercise, aerobic exercise was performed using a static bicycle where the patient cycled at 50 rotation per minute (RPM) and load KP 0 for 15 minutes for week I, 30 minutes for week II, 45 minutes for weeks III-IV, and 60 minutes for week $\mathrm{V}$. Load was increased to $1 \mathrm{KP}$ for 15 minutes for week VI, 30 minutes for week VII, and 45 minutes for week VIII. For strengthening exercises, the load was increased according to the exercise test results ( $50 \%$ for ten maximum repetitions), for five repetitions, hold for 6 seconds, in three sets for each muscle. In the aquatic exercise group, the intervention was done in a $3 \mathrm{~m} \times 3 \mathrm{~m} \times 1.2$ $\mathrm{m}$ pool with $32^{\circ} \mathrm{C}$ water. Ankle bands, which were used in the intervention, were accessories from the pool and has been standardized. Since there was no adjustment in height for subjects, we assumed their height in the water was similar, approximately at the level of the xiphoid process. Aerobic exercise was done by making a quick movement in four directions (forward, backward, left, and right) in one repetition in week I, two repetitions in week II, three repetitions in weeks III and IV, four repetitions in weeks V and VI, and five repetitions in weeks VII and VIII. Strengthening exercise was done using ankle weights in three sets for each muscle for six repetitions, hold for 6 seconds in weeks I-IV, then increasing repetitions by ten in weeks V-VIII. Training was performed by a certified physiotherapist who guided the exercise.

Cardiorespiratory endurance was assessed before and after exercise 3 times per week for eight weeks. We used the Borg scale and the Borg CR-10 scale, which consisted of rating perceived exertion (RPE), Borg scale dyspnea index (BSDI), and leg fatigue (LF) parameters. Stress testing was done to assess cardiorespiratory endurance using the Astrand Test 3 times (before the intervention, after four weeks of intervention, and after the last intervention). For quality of life assessment, The Short Form 36 Health Survey Questionnaire (the SF-36) was used. The SF36 consists of 36 questions that represent the following eight domains: physical function (PF), role limitations due to physical health (RLPH), role limitations due to emotional problems (RLEP), energy fatigue (EF), emotional well-being (EWB), social functioning (SF), pain, and general health $(\mathrm{GH})$.

\section{Ethics}

The Research Ethics Committee of the Faculty of Medicine, Universitas Indonesia-Cipto Mangunkusumo Hospital has approved the study protocol (803/UN2.F1/ETIK/X/2016).

\section{Statistical analysis}

In this study, data were analyzed using Statistical Product and Service Solutions (SPSS) version 20.0 (IBM Corp., New York, USA) with an independent t-test, Wilcoxon, and Mann-Whitney tests, if necessary.

\section{RESULTS}

Of the 41 participants, 33 individuals completed this study. The calculated sample size was not achieved because six subjects did not complete the exercise and two subjects discontinued (Figure 1). The reasons were unexpected events such as a traumatic event independent of the intervention, a subject returned to her hometown, and hospitalization because of disease unrelated to the intervention. Demographic data and characteristics of study subjects are similar to each group (Table 1 ).

The results of this study showed there were no statistically significant differences in cardiorespiratory endurance improvement between the aquatic exercise group and the land-based exercise group. The assessment of cardiorespiratory endurance in land-based exercise after eight weeks of intervention using 


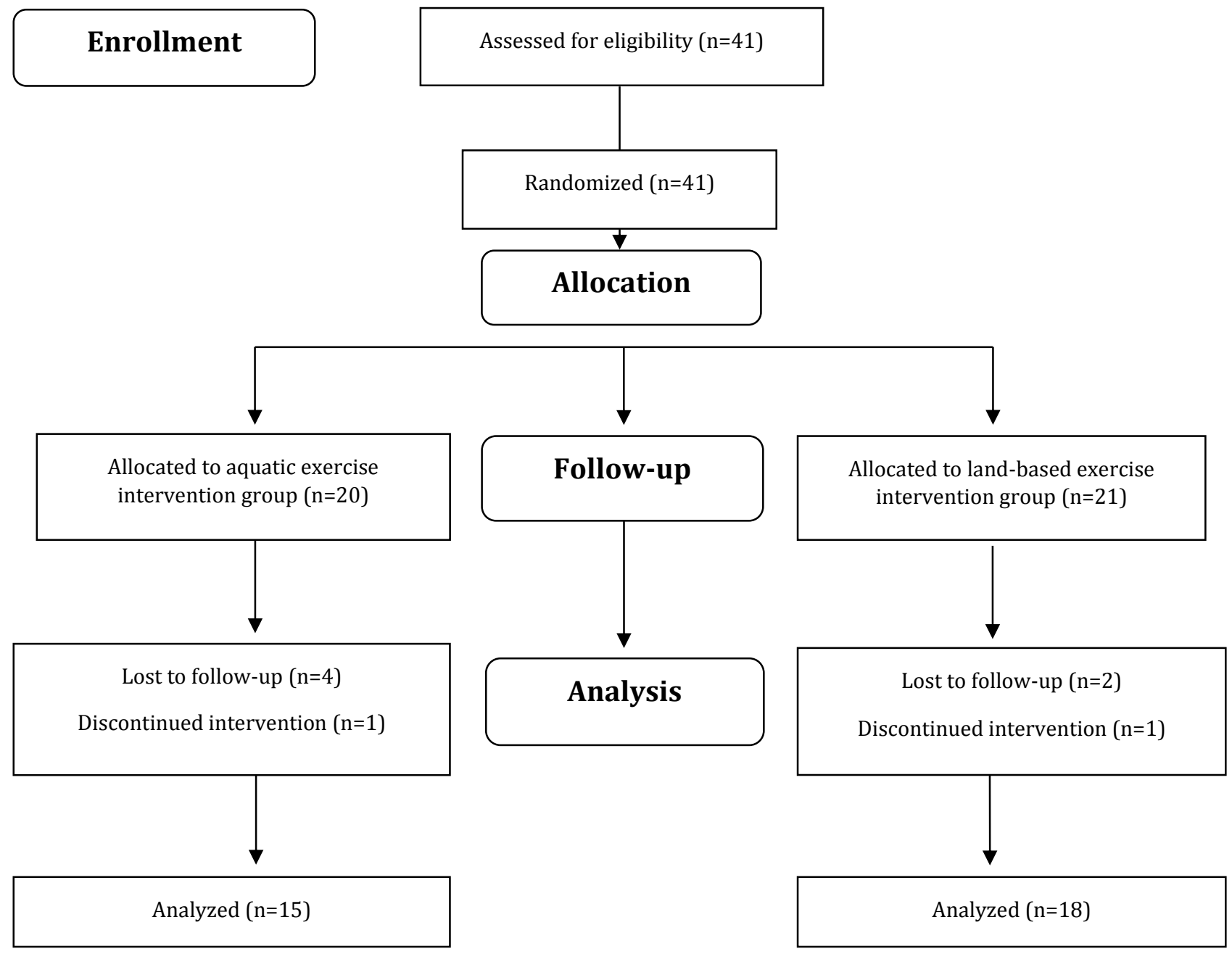

Figure 1. Flowchart of the study protocol

the Borg scale showed a significant increase in the RPE parameter $(p=0.02)$, rather than in BSDI $(p=0.719)$ and LF $(p=0.427)$. There was also an increase in cardiorespiratory endurance in the LF parameter $(\mathrm{p}=0.016)$ rather than in rating RPE $(p=0.383)$ and BSDI $(p=0.391)$ after intervention in the aquatic exercise group. However, when both interventions were compared, there were no significant differences in RPE $(p=0.770)$, BSDI $(\mathrm{p}=0.877)$, and LF $(\mathrm{p}=0.274)$.

Quality of life assessment using the SF-36 form showed a statistically significantimprovement in the land-based exercise group after eight weeks. Improvements are shown in the RLPH $(\mathrm{p}=0.024)$, $\operatorname{RLEP}(\mathrm{p}=0.041)$, and EF ( $\mathrm{p}=0.016)$ domains, but there was a decrement in the pain $(p=0.049)$ domain (Table 2). In the aquatic exercise group, there were improvements in EF $(p=0.025), E W B$ $(p<0.001)$, and GH ( $p=0.045)$ domains (Table 2). In comparison, there were no statistically significant differences in quality of life improvement between the aquatic exercise group and the land-based exercise group in each domain (Table 2).

\section{DISCUSSION}

Knee $\mathrm{OA}$ is a major cause of arthritisrelated disability, limiting daily activities not only reduces the quality of life for individuals. ${ }^{9}$ Strengthening exercise can both increase muscle strength and maintain muscle mass, while aerobic exercise is aimed at relieving pain and improving knee function. Both exercises can reduce pain, improve physical performance, and prevent disability. ${ }^{9,10}$

When prescribing effective exercises, cardiorespiratory endurance measurements 
Table 1. Baseline characteristics of participants in both study groups

\begin{tabular}{|c|c|c|}
\hline Characteristics & $\begin{array}{c}\text { Aquatic } \\
\text { exercise } \\
\mathrm{n}(\%) \text { or } \\
\text { mean (SD) } \\
(\mathrm{n}=15)\end{array}$ & $\begin{array}{c}\text { Land-based } \\
\text { exercise } \\
n(\%) \text { or } \\
\text { mean (SD) } \\
(n=18)\end{array}$ \\
\hline Age (years) & $61(42.72)$ & $64.5(48.74)$ \\
\hline Body weight (kg) & $68(57.86)$ & $69.5(55.11)$ \\
\hline $\begin{array}{l}\text { Waist circumference } \\
(\mathrm{cm})\end{array}$ & $93(75.10)$ & $96.5(76.11)$ \\
\hline \multicolumn{3}{|l|}{ Occupation } \\
\hline Housewife & $15(100.00)$ & $14(77.78)$ \\
\hline Employee & $0(0)$ & $4(22.22)$ \\
\hline \multicolumn{3}{|l|}{ Education } \\
\hline High school & $9(60.00)$ & $5(27.70)$ \\
\hline Diploma & $3(20.00)$ & $3(16.67)$ \\
\hline Graduate & $3(20.00)$ & $10(55.56)$ \\
\hline \multicolumn{3}{|l|}{ Comorbid condition } \\
\hline Hypertension & $11(73.30)$ & $14(77.80)$ \\
\hline Dyslipidemia & $5(33.30)$ & $6(33.30)$ \\
\hline Diabetes mellitus & $1(6.7)$ & $2(11.10)$ \\
\hline Metabolic syndrome & $5(33.3)$ & $5(27.80)$ \\
\hline Onset of knee OA (years) & $3.3(0.70)$ & $2.6(0.4)$ \\
\hline Onset of obesity (years) & $12(2.40)$ & $11.9(1.90)$ \\
\hline \multicolumn{3}{|l|}{ Nutritional status } \\
\hline Obesity I & $7(46.70)$ & $6(33.30)$ \\
\hline Obesity II & $8(53.30)$ & $12(66.70)$ \\
\hline
\end{tabular}

$\mathrm{SD}=$ standard deviation; $\mathrm{OA}=$ osteoarthritis

are required. Measurement of cardiorespiratory endurance can be observed objectively from maximal oxygen consumption $\left(\mathrm{VO}_{2} \max \right) \cdot \mathrm{VO}_{2} \max$ is measured in millimeters of oxygen used in one minute per kilogram of body weight $(\mathrm{ml} / \mathrm{kg} / \mathrm{min})$. It is establish the maximum amount of oxygen a person can utilize during exercise. The Borg scale (RPE) correlates with actual heart rate during physical activity; thus, it could measure $\mathrm{VO}_{2}$ max. RPE on sensations felt during physical activity, takes into account personal physical fitness level and general fatigue levels. ${ }^{11}$ In addition to RPE, breathlessness and leg fatigue are considered influential to determine energy expenditure. To accommodate the condition (such as patient with muscle fatigue which contributes substantially to the limited exercise capacity, etc), this study utilizes the Borg CR-10 scale, which consists of dyspnea (BSDI) and leg fatigue components, as a modification of the Borg scale. Breathlessness is nota simple one-dimensional symptom; it consists of both sensory and affective aspects, such as effort and speed exercise. In fact, breathlessness is one of the factors that cause a person to stop doing an exercise. ${ }^{12}$ Leg fatigue, on the other hand, is related to heart rate, blood lactate concentration, and respiratory rate. ${ }^{13}$ The Borg scale can also be used to assess cardiorespiratory endurance. ${ }^{12}$

\section{Effects of aquatic exercise and land-based exercise on cardiorespiratory endurance in obese patients with knee osteoarthritis}

In this study, there were no statistically significant differences in cardiorespiratory endurance improvement between the aquatic exercise group and the land-based exercise group in each domain. Similar with many recent studies, for example Lieber et al $^{14}$ compared the effects of an 11.5-weeks running or swimming program for men who trained three times/week at an HR intensity of $75 \%$ of $\mathrm{VO}_{2}$ max, and no significant difference in the $\mathrm{VO}_{2}$ max was found between running and swimming groups. ${ }^{14}$ Wilber et $\mathrm{al}^{15}$ compared the effects of 6-weeks deep water running to treadmill running at similar intensities (90-100\% and $\left.70-75 \% \mathrm{VO}_{2} \max \right)$ on $\mathrm{VO}_{2} \max$ and no significant differences were observed between programs. ${ }^{15}$ Taunton et $\mathrm{al}^{16}$ evaluated the effectiveness of a generalized water-based exercise program compared to a land-based exercise program on cardiorespiratory fitness of women aged 65-75 years. At the end of the programs, no significant differences in cardiorespiratory fitness were observed between land-based exercise and water-based exercise groups. ${ }^{16}$

Meanwhile, the results of this study are different from those of Bocalini et $\mathrm{al}^{17}$ in which aquatic exercise was found to further enhances cardiorespiratory endurance than land-based exercise. Fifty healthy sedentary women were randomly assigned to aquatic exercise and landbased exercise. The two groups were exercised for 12 weeks the aquatic exercise and land-based exercise increased $\mathrm{VO}_{2}$ max by $42 \%$ (before, $20 \pm 3$; after, $35 \pm 3 \mathrm{ml} / \mathrm{kg}$ per $\mathrm{min}$ ) and $32 \%$ (before, $19 \pm 4$; after, $28 \pm 2 \mathrm{ml} / \mathrm{kg}$ per $\mathrm{min}) \cdot{ }^{17}$ The differences with our study might be due to type and duration of the intervention. In the study of Bocalini et al, ${ }^{17}$ intervention in the aquatic exercise group was a variation of resistance exercise. Whereas in the land-based exercise group, subjects only 
Table 2. Quality of life comparison between the aquatic exercise group and the land-based exercise group

\begin{tabular}{|c|c|c|c|c|c|c|c|}
\hline \multirow{2}{*}{ SF-36 } & \multicolumn{3}{|c|}{ Aquatic exercise } & \multicolumn{3}{|c|}{ Land-based exercise } & \multirow{2}{*}{$\begin{array}{l}\text { p-value } \\
\text { between } \\
\text { group }\end{array}$} \\
\hline & Pre* & Post* & $\mathrm{p}$ value & Pre* & Post* & $\mathrm{p}$-value & \\
\hline $\begin{array}{l}\text { Physical function } \\
\text { (PF) }\end{array}$ & $50(15,85)$ & $50(15,80)$ & 0.523 & $60(15,100)$ & $75(25,100)$ & 0.06 & 0.324 \\
\hline $\begin{array}{l}\text { Role limitations } \\
\text { due to physical } \\
\text { health (RLPH) }\end{array}$ & $50(0,100)$ & $62.5(0,100)$ & 0.385 & $62.5(0,100)$ & $100(0,100)$ & 0.024 & 0.229 \\
\hline $\begin{array}{l}\text { Role limitations } \\
\text { due to emotional } \\
\text { problems (RLEP) }\end{array}$ & $100(0,100)$ & $100(0,100)$ & 0.226 & $100(0,100)$ & $100(33.3,100)$ & 0.041 & $0.062^{\dagger}$ \\
\hline $\begin{array}{l}\text { Energy/fatigue } \\
\text { (EF) }\end{array}$ & $65(50,80)$ & $75(50,90)$ & 0.025 & $65(39,90)$ & $72.5(50,100)$ & 0.016 & 0.593 \\
\hline $\begin{array}{l}\text { Emotional well- } \\
\text { being (EWB) }\end{array}$ & $68(52,96)$ & $88(72,100)$ & $<0.001$ & $82(56,100)$ & $86(64,100)$ & 0.140 & 0.058 \\
\hline $\begin{array}{l}\text { Social function } \\
\text { (SF) }\end{array}$ & $87.5(62.5,100)$ & $75(50,100)$ & 0.440 & $87.5(35.5,100)$ & $100(50,100)$ & 0.180 & $0.093^{\dagger}$ \\
\hline Pain & $57.5(32.5,77.5)$ & $57.5(32.5,100)$ & 0.936 & $57.5(32.5,70)$ & $67.5(20,100)$ & 0.049 & $0.062^{\dagger}$ \\
\hline $\begin{array}{l}\text { General health } \\
\text { (GH) }\end{array}$ & $60(20,75)$ & $65(40,75)$ & 0.045 & $65.5(25,80)$ & $70(30,85)$ & 0.119 & 0.613 \\
\hline
\end{tabular}

*Data are presented in median (min,max); ${ }^{\dagger}$ Mann-Whitney test

performed walking exercises. This difference could cause an imbalance in the results. ${ }^{17}$

This study showed that there was a significant potential of aquatic and landbased exercise in improving cardiorespiratory endurance, although each other improved in different parameters. There was an increase in cardiorespiratory endurance as indicated by the RPE parameter in the land-based exercise group. These findings were similar to those of Larose et $\mathrm{al}^{10}$ who reported that aerobic exercise on land could improve cardiorespiratory endurance in obese patients with knee OA. Cardiorespiratory endurance was measured by $\mathrm{VO}_{2}$ peak and improved in both female walkers $(4 \% ; \mathrm{p}<0.001)$ and male walkers $(5 \% ; \mathrm{p}<0.001)$. The prescribed intervention was supervised walking exercise by trained medical personnel three times per week for six months. ${ }^{10}$

For the aquatic exercise group, there was a significant increase in the LF parameter compared with the other two parameters. This result is different with Wouters et $\mathrm{al}^{18}$ who reported no significant difference on the Borg CR-10 after administering aquatic exercise $(\mathrm{p}=0.11)$. Aquatic exercise consists of heating, running while in the water, and cooling down accompanied by music stimulation during the program. It comprises 12 one-hour sessions, twice per week for six weeks. ${ }^{18}$ The differences between the results were due to type and duration of the intervention. In the Wouters et $\mathrm{al}_{1}{ }^{18}$ intervention in the aquatic exercise group subjects only performed walking exercises for six weeks meanwhile in this study we have used ankle weights for eight weeks.

This study showed that aquatic exercise could reduce muscle fatigue. Aquatic exercise has been reduced weight-bearing stresses on the skeletal joints and to provide therapeutic benefits in conditions of orthopedic troubles. Also, another potential to improve cardiovascular fitness given that water has 700 times the density of air. This promotes an increase of energy expenditure for work done, and a reduction of the impact on the joints and partial weight support is provided by the buoyancy of water. ${ }^{17}$

The warm environment of water and hydrostatic pressure can reduce edema, the 
burden of painful joints, and also decrease muscle spasms, leading to muscle relaxation. It was also mentioned that warm temperature could decrease the activity of creatine kinase in the blood, which is associated with muscle fatigue as well as muscle damage caused by exercise. ${ }^{8,19}$ Under warm conditions, blood flow to muscles will increase, and there will be a reduction of mediator molecules that activate nociceptors, resulting in reduction of pain, and inducing muscle relaxation. ${ }^{20}$

Local muscle fatigue and central dyspnea are considered the two most important factors in healthy people in addition to perceived exertion. A strong relationship exists between RPE and both factors that can be illustrated with improved work function. They can cause a cessation of physical exercise when a person passes a tolerable level. ${ }^{12}$

Our studies indicate that both aquatic exercise and land-based exercise have advantages. The buoyancy effect and warm water immersion during exercise could increase heat shock proteins that could protect muscles from damage due to exercise. ${ }^{17}$ Land-based exercise can increase muscle strength, therefore, it could improve knee function in knee OA patients. Increasing the level of physical activity in knee OA patients is the only way to improve cardiorespiratory endurance and functional status. ${ }^{10}$

Based from the results, it would be beneficial if obese patients with knee OA who had subacute pain (VAS ranging 4-6) taking exercise under lukewarm water environment for the beginning to avoid leg fatigue and help relax muscle and reduce pain. ${ }^{11}$ Then after there are body adaptation to exercise and stronger muscles, the patients could be encouraged to have land-based exercise session as it would improve cardiorespiratory endurance.

\section{Effects of aquatic exercise and land-based exercise on quality of life in obese patients with knee osteoarthritis}

This study showed that aquatic and land-based exercise could also improve quality of life obese patients with knee osteoarthritis. In this study, there were no significant differences between aquatic exercise and land-based exercise, as another study conducted by Lim et $\mathrm{al}^{21}$ had the same results. SF-36 was used in this study because it has already widely used and easily self-administered in physical activity research. SF-36 is commonly used to provide an indication of the health status of particular populations, to help with service planning and to measure the impact (in terms of health gains) of clinical and social interventions. The SF-36 is a 36 item scale, which measures eight domains of health status: physical functioning, physical role limitations, emotional role limitations, energy/ fatigue, emotional well-being, social functioning, pain, and general health. ${ }^{22}$

In this study, there was no significant difference between the aquatic exercise group and the land-based exercise group in all domains. Lim et $\mathrm{al}^{21}$ reported that an increase in compliance in aquatic exercise might reduce joint pressure and heavy feeling during movement in water, decrease the sweating effect, reduce stiffness because of the warm temperature of the water, reduce pain, and improve functioning compared with analogous parameters in land-based exercise. ${ }^{21}$ Suomi et $\mathrm{al}^{23}$ also reported that, in patients with arthritis, both aquatic and land-based exercise have a positive effect on activity of daily living and physical function after 8 weeks, but found no significant difference between the 2 programs. ${ }^{23}$

In land-based exercise group, there were quality of life improvements in the RLEP, RLPH, and EF domains accompanied by a decrease in the pain domain. These results were similar those stated in Kao et $\mathrm{al}^{24}$ study. In 2012, Kao et $\mathrm{al}^{24}$ conducted a study in 205 knee OA patients who were divided into two groups: a landbased exercise group and a control group for eight weeks. The differences between this study compared to Kao et $\mathrm{al}^{24}$ are we used strengthening and aerobic exercise while they focused on lower limb stretching and strengthening.

Obese patients with knee OA often avoid physical activities due to pain and frailty. As aerobic exercise improve biomechanical gait, maintaining body weight, improve balance and reduce disability and pain, patients could do daily activities feasibly. Due to sarcopenia and associated muscle weakness which is often found in this population, strengthening exercise has been proven to improve function, strength and pain in knee OA condition. Thus, combination of these exercises might improve limitations that affects 
this population. ${ }^{4}$ As the subjects were given aerobic and strengthening exercise, they tend to have less depressive symptoms and gain confidence. Recent studies showed that aerobic exercise changed gene expressions in locus coeruleus and dorsal raphe nucleus to make people more resilience dealing with stress and depression. ${ }^{9}$

Beside the benefits of land-based exercise, aquatic exercise could also increase quality of life in obese patients with knee $\mathrm{OA}$, especially mental component. In this study, there were significant improvements in energy/fatigue, emotional wellbeing (mental component summary), and general health (physical component summary) domains. In 2010, Lim et al ${ }^{21}$ conducted a study in 75 obese patients with knee OA. Subjects were divided into three groups, the aquatic exercise group, the landbased exercise group, and the control group. There were significant improvements in the mental component summary and the physical component summary of the SF-36. Because obese patients with knee OA may experience both pain and discomfort during exercise, it is important for practitioners to develop exercise therapies that could help to reduce pain during exercise. ${ }^{25}$ Physical exercise in water is a possibility to try to increase physical and mental health of obese persons without the risk of injuries. ${ }^{18}$ It has been reported that aquatic exercise effects not only weight reduction and muscle strengthening, but also pain relief; and its effect extends to enhancing the quality of life. ${ }^{21}$ This could be good evidence for applying an exercise program to patients who need to do exercise but fail to comply because of increased pain. ${ }^{21}$ After the aquatic exercise, the health-related quality of life scores of participants showed an improvement in public distress and in the scales that normally deviate most from normal weight people: physical function and self-esteem. ${ }^{18}$ Furthermore, Nagle et $\mathrm{al}^{6}$ have investigated in forty-four obese patients undergoing 16-weeks intervention shown the aquatic exercise group had slightly greater nonsignificant improvements on four out of seven SF-36 domains (role physical, bodily pain, general health, and social functioning) than the land-based group. The proposed mechanism underlying this response is an increased sensory input and muscle relaxation from the hydrodynamic and thermoregulatory properties of water. As a result of this increased physical and mental stimulation, it has been suggested that aquatic exercise distracts individuals from pain sensation.
A limitation of this study was that the sample size of this study in small population, however, was used randomized controlled trial. Other limitations are there is no follow-up procedure was performed to observe the aftereffects of exercise.

In conclusion, for the clinical settings it can be recommended that obese patients with knee OA with subacute pain start aquatic exercise due to the buoyancy and lukewarm water environment effect to reduce leg fatigue and to prevent injury due to accumulated stress on knee joint for improving cardiorespiratory fitness and enhanced general health, mental health and energy to consider altering the model of the exercise. After that exercise could be continued in land-based settings to improve RPE, RLPH, RLEP and decreased pain, which is important to improve knee OA conditions. This method has been done as a service protocol in the Obesity Outpatient Clinic Medical Rehabilitation Department of Cipto Mangunkusumo Hospital.

\section{Conflict of Interest}

The authors affirm no conflict of interest in this study.

\section{REFERENCES}

1. Flier J, Maratos S. Biology of obesity . In: Fauci SA, Braunwald E, Kasper DI, Hauser SL, Longo DL, Jameson JL, et al, editors Harrison's principles of internal medicine. 17th ed. USA: McGraw to Hill; 2008. 462-8. p.

2. Riskesdas. Riset Kesehatan Dasar. Badan Penelitian Dan Pengembangan Kesehatan Kementrian Kesehatan RI. 2013. p. 223-30. Indonesian.

3. Sitik T, Stiskal D, Foye P, Nadler R, Wyss J, Heslop S. Osteoarthritis. In: Frontera WR, editor. DeLisa's physical medicine and rehabilitation: principles and practice. 5th ed. Philadelphia: Lippincott Williams \& Wilkins; 2010. 781-809 p.

4. Messier SP. Diet and exercise for obese adults with knee osteoarthritis. Clin Geriatr Med. 2010;26(3):461-77.

5. Boidin M, Lapierre G, Paquette Tanir L, Nigam A, Juneau $\mathrm{M}$, Guilbeault V, et al. Effect of aquatic interval training with Mediterranean diet counseling in obese patients: results of a preliminary study. Ann Phys Rehabil Med. 2015;58(5):269-75.

6. Nagle EF, Robertson RJ, Jakicic JJ, Otto AD, Ranalli JR, Chiapetta LB. Effects of aquatic exercise and walking in sedentary obese women undergoing a behavioral weight-loss intervention. Int J Aquatic Res Educ. 2007;1:43-56.

7. Vinod Kumar KC, Regima SE, Vikranth GR. Effectiveness of land based endurance training versus aquatic based 
endurance training on improving endurance in normal individuals. Int J Physiother. 2015;2(3):466-71.

8. de Mattos F, Leite N, Pitta A, Bento PC. Effects of aquatic exercise on muscle strength and functional performance of individuals with osteoarthritis: a systematic review. Rev Bras Reumatol Engl Ed. 2016;56(6):530-42.

9. Semanik PA, Chang RW, Dunlop DD. Aerobic activity in prevention and symptom control of osteoarthritis. PM R. 2012;4(5):S37-44.

10. Larose J, King J, Brosseau L, Wells GA, Reid R, Maetzel A, et al. The effect of walking on cardiorespiratory fitness in adults with knee osteoarthritis. Appl Physiol Nutr Metab. 2013;38(8):886-91.

11. Pescatello LS, Arena R, Riebe D, Thompson DP. ACSM's guidelines for exercise testing and prescription. 9th ed. China: Wolters Kluwer; 2014. p. 72-86.

12. Borg E, Borg G, Larsson K, Letzter M, Sundblad BM. An index for breathlessness and leg fatigue. Scand J Med Sci Sports. 2010;20(4):644-50.

13. Alnahdi AH, Zeni JA, Snyder-Mackler L. Muscle impairments in patients with knee osteoarthritis. Sports Health. 2012;4(4):284-92.

14. Lieber DC, Lieber RL, Adams WC. Effects of runtraining and swim-training at similar absolute intensities on treadmill V02max. Med Sci Sports Exerc. 1989;21(6):655-61.

15. Wilber RL, Moffatt RJ, Scott BE, Lee DT, Cucuzzo NA. Influence of water run training on the maintenance of aerobic performance. Med Sci Sports Exerc. 1996;28(8):1056-62.

16. Taunton JE, Rhodes EC, Wolski LA, Donelly M, Warren J, Elliot J et al. Effects of land-based and water-based fitness programs on the cardiovascular fitness, strength and flexibility of women aged 65-75 years. Gerontology. 1996;42(4):204-10.
17. Bocalini DS, Serra AJ, Murad N, Levy RF. Water- versus land-based exercise effects on physical fitness in older women. Geriatr Gerontol Int. 2008;8(4):265-71.

18. Wouters EJ, Van Nunen AM, Geenen R, Kolotkin RL, Vingerhoets AJ. Effects of aquajogging in obese adults: a pilot study. J Obes. 2010;2010:231074.

19. Skurvydas A, Kamandulis S, Stanislovaitis A, Streckis V, Mamkus G, Drazdauskas A. Leg immersion in warm water, stretch-shortening exercise, and exercise-induced muscle damage. J Athl Train. 2008;43(6):592-9.

20. Lund H, Weile U, Christensen R, Rostock B, Downey A, Bartels EM, et al. A randomized controlled trial of aquatic and land-based exercise in patients with knee osteoarthritis. J Rehabil Med. 2008;40(2):137-44.

21. Lim JY, Tchai E, Jang SN. Effectiveness of aquatic exercise for obese patients with knee osteoarthritis: a randomized controlled trial. PM R. 2010;2(8):723-31.

22. Burholt V, Nash P. Short form 36 (SF-36) health survey questionnaire: normative data for Wales. J Public Health. 2011;33(4):587-603.

23. Suomi R, Collier D. Effects of arthritis exercise programs on functional fitness and perceived activities of daily living measures in older adults with arthritis. Arch Phys Med Rehabil 2003;84(11):1589-94.

24. Kao MJ, Wu MP, Tsai MW, Chang WW, Wu SF. The effectiveness of a self-management program on quality of life for knee osteoarthritis (OA) patients. Arch Gerontol Geriatr. 2012;54(2):317-24.

25. Bartels EM, Lund H, Hagen KB, Dagfinrud H, Christensen R, Danneskiold-Samsøe B. Aquatic exercise for the treatment of knee and hip osteoarthritis. In: The Cochrane Collaboration, editor. Cochrane Database of Systematic Reviews [Internet]. Chichester, UK: John Wiley \& Sons, Ltd; 2007 [cited 2018 Jun 26]. Available from: http://doi. wiley.com/10.1002/14651858.CD005523.pub2 